\title{
23. ON THE STRUCTURE AND CHEMICAL COMPOSITION OF METEOR BODIES OF COMETARY ORIGIN
}

\author{
E. N. KRAmER \\ (University Observatory, Odessa, U.S.S.R.)
}

\begin{abstract}
A BST RACT
Possible origins for hyperbolic meteor orbits are noted, and photographic evidence on the nature of meteoroid structure is discussed.

The spectral investigation of meteors permits us to find out what chemical elements are contained in meteoric bodies of cometary origin. However, this cannot give a complete idea about the true chemical composition of meteoric bodies. The appearance of any lines in a meteor spectrum depends not only on the chemical composition of the body, but also on the excitation conditions of the atomic levels producing these lines. Indirect data about the structure and the chemical composition of meteoric bodies of cometary origin can be obtained by studying the velocity, deceleration and luminosity of meteors. Below we shall give some results of the investigation of meteor events, permitting us, to a certain degree, to decide about the nature of meteoric bodies having a cometary origin.
\end{abstract}

1. At various times many authors, by reducing meteor photographs, have found hyperbolical orbits for meteors. It is clear that the hyperbolic elements of many of them are the consequence of errors of measurement. However, the deviations of the heliocentric velocity of several meteors from the parabolic limit cannot be interpreted as occasional errors of measurement. Such results were obtained both in the Soviet Union and in Czechoslovakia. The hyperbolical orbits were discovered particularly for the meteors with all the elements of the orbits (apart from $a$ and $e$ ) very near to those of the Perseids. It can be concluded from this that these meteors become hyperbolic inside the solar system.

We have studied the conditions of approach to the planets of observed meteoroids having hyperbolic orbits. It was found in these cases that the change of the original elliptical orbit into a hyperbolic one could not be explained by gravitational perturbations of the planets. Then we put forward, in 1963, an assumption that disintegration of comparatively large meteoric bodies had taken place not long before the appearance of the meteor. As a result of the velocity of the fragment's ejection we can observe both comparatively slow and comparatively fast meteors. The former produce short- 
period orbits inside the meteor stream, the later produce hyperbolic orbits. In such a way the existence of hyperbolic meteors indicates the possibility of the fragmentation of cometary meteoroids before the beginning of radiation, and this fragmentation is of the nature of an explosion.

2. In 1958 a photograph of a bright meteor was obtained at the Odessa Astronomical Observatory. During its flight this meteor produced several flares. Using the rotating-shutter intervals the velocity was calculated at different points of the meteor path. It was established that this meteor moved with acceleration, and only near the end of its trajectory was the ordinary deceleration detected. We have traced the curves showing the changes of velocity separately for the beginnings and the ends of the rotating-shutter breaks. The first curve is normal, the meteor decelerates at first slowly and then rapidly. But the second curve, traced from the ends of breaks, shows an acceleration. This type of variation in the velocity could be explained by a fragmentation of the meteoroid, with some fragments receiving an impulse directed forward.

3. On August 8,1965 , in Odessa a photograph of a bright meteor was obtained with the velocity at the beginning of the path $20 \mathrm{~km} / \mathrm{sec}$. At the height of $73 \mathrm{~km}$ the meteoric body disintegrated into two parts. The fragmentation was in the form of an explosion, the velocity of one of the fragments being $1.37 \pm 0.30 \mathrm{~km} / \mathrm{sec}$ greater and of the other $1.28 \pm 0.30 \mathrm{~km} / \mathrm{sec}$ smaller than the velocity of the parent body. At a height of $72 \mathrm{~km}$ the velocity of the first fragment was equal to $20.9 \mathrm{~km} / \mathrm{sec}$, i.e. it moved more rapidly than the parent body at the moment of its entry into the dense layers of the terrestrial atmosphere, corresponding to the beginning of intensive evaporation. Fragmentation, and the comparatively great relative velocities of the fragments, were found by Ceplecha in $1953(800 \mathrm{~m} / \mathrm{sec})$ and by the author for the fireball of May 11 , $1955(200 \mathrm{~m} / \mathrm{sec})$.

The data mentioned above permit us to put forward the following hypothesis. Various meteoric bodies of cometary origin contain inclusions that can, under certain
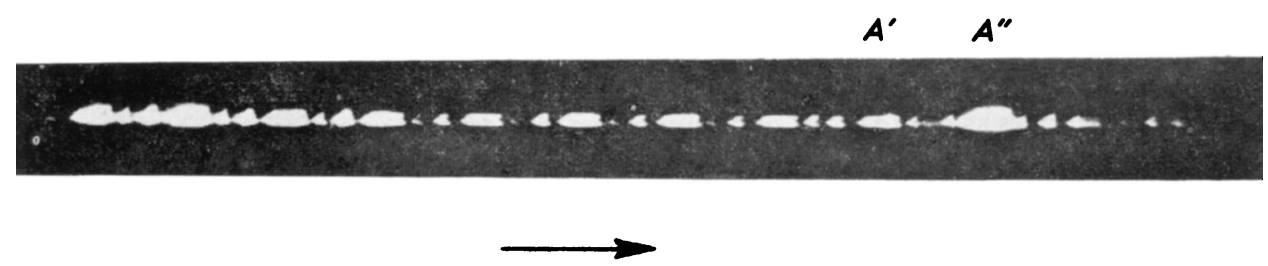

FIG. 1. The image of a meteor photographed on August 8, 1965, through a rotating shutter with three different open sections. The triangular form of the segments of the meteor path is due to diffraction effects. Note that the distances between the fainter and the mean segment are almost constant up to the point $A^{\prime}$, but then this distance increases instantaneously. A new fragment has appeared between $A^{\prime}$ and $A^{\prime \prime}$, and after the point $A^{\prime \prime}$ it becomes bright. This means the division of the body into two objects. 


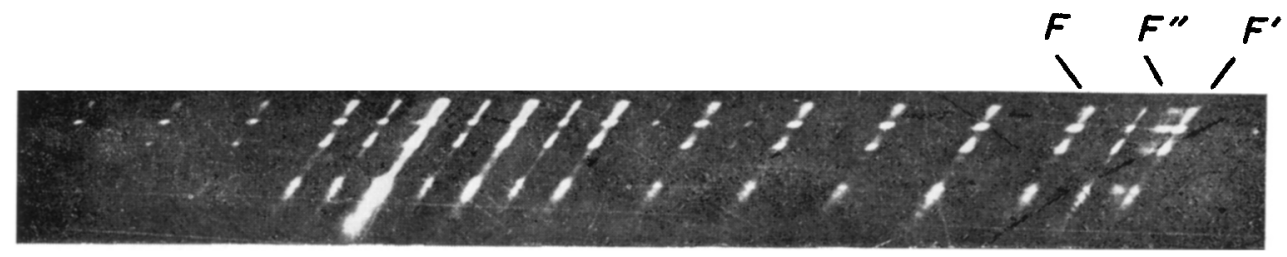

FIG. 2. The spectrum of the meteor of August 8, 1965, photographed through a shutter with two apertures, $5^{\circ}$ and $30^{\circ}$. In the middle of the path the short exposure was not long enough to give an image. At the end of the path we can see the separation of a small fragment.

conditions, lead to the explosion and fragmentation of the meteoroid. Such a fragmentation can occur both in the Earth's atmosphere (as in the case of the meteor of 1965) and outside its limits (as in the cases of hyperbolic orbits). These phenomena show that the inclusions must be sufficiently volatile. We hope that the spectrograms of meteors taken with great dispersion, as well as the direct study in the laboratory of meteoric particles of cometary origin trapped by rockets, will permit us to find the chemical nature of these inclusions.

\section{DISCUSSION}

Whipple: A relative velocity of $1 \mathrm{~km} / \mathrm{sec}$ requires extraordinarily great energy of the explosion. I suggest that other interpretations of the measurements are possible. For example, the meteoroid may have split prior to observation, and intensity variations in the components may account for the apparent acceleration. The relative velocity could produce a lateral motion giving a $6^{\circ}$ angle in a split meteor. I have never observed a split meteor with a separation angle exceeding $20^{\prime}$.

Sekanina: Should the fragmentation of meteoroids be understood as a property of the body itself rather than a consequence of the action of the Earth's atmosphere? The velocity of separation of several hundreds of metres per second seems to me rather high, especially in comparison with the splitting velocities of comet nuclei (several m/sec, exceptionally $20 \mathrm{~m} / \mathrm{sec}$ ).

Kramer: The fragmentation of a meteor body is affected by the internal forces.

Levin: There are two different problems: one of the high velocities of fragments originating when the fragmentation occurs in the atmosphere, and the other - of the fragmentation in space suggested by Kramer, which produces the hyperbolic and short-period orbits among the Perseids. The first one may perhaps be correct, and if so, we must look for some explanation of these 'explosions'. However, in respect of the second problem I completely disagree with Kramer. I regard the excessive heliocentric velocities as due to errors of determination of geocentric velocities.

Kramer: I think that the hyperbolic velocity of some meteors is real.

Whipple: I agree with Dr. Levin. If hyperbolic velocities are real, some should appear at much smaller geocentric velocities, not always near the upper limit of $72 \mathrm{~km} / \mathrm{sec}$.

Lindblad: Have you determined the orbital elements of the May 1955 fireball?

Kramer: The orbit of the 1955 fireball has not been calculated. 